OPEN ACCESS

Edited by:

Letian Dou,

Purdue University, United States

Reviewed by:

Jingjing Xue,

University of California, Los Angeles,

United States

Yongsheng Liu,

Nankai University, China

${ }^{*}$ Correspondence:

Hang Zhou

zhouh81@pkusz.edu.cn

Specialty section:

This article was submitted to

Solar Energy,

a section of the journal

Frontiers in Energy Research

Received: 01 April 2021

Accepted: 24 May 2021

Published: 02 July 2021

Citation:

Zhong H, Zhou R, WuX, Lin X, Wang Y, $L i Q$ and Zhou $H$ (2021) Investigation of the S-Shaped Current-Voltage Curve in High Open-Circuit Voltage Ruddlesden-Popper Perovskite

Solar Cells.

Front. Energy Res. 9:689657. doi: 10.3389/fenrg.2021.689657

\section{Investigation of the S-Shaped Current-Voltage Curve in High Open-Circuit Voltage Ruddlesden-Popper Perovskite Solar Cells}

\author{
Hong Zhong, Renlai Zhou, Xiaoqing Wu, Xiaoyun Lin, Ya Wang, Qian Li and Hang Zhou*
}

School of Electronic and Computer Engineering, Peking University Shenzhen Graduate School, Shenzhen, China

We report our investigation on the S-shaped current-voltage characteristics in a hotcasting-processed $(\mathrm{BA})_{2}(\mathrm{MA})_{3} \mathrm{~Pb}_{4} \mathrm{l}_{13}$ Ruddlesden-Popper (RP) perovskite solar cell. The two-dimensional perovskite solar cells are fabricated with $\mathrm{NiO}_{x}$ as the hole transport layer $(H T L)$, which leads to significantly high open-circuit voltage $\left(V_{o c}\right)$. The champion device shows a $V_{\text {oc }}$ of $1.21 \mathrm{~V}$ and a short current density $\left(\mathrm{J}_{\mathrm{sc}}\right)$ of $17.14 \mathrm{~mA} / \mathrm{cm}^{2}$, leading to an overall power conversion efficiency (PCE) of 13.7\%. Although the PCE is much higher than the control device fabricated on PEDOT:PSS, a significant S-shaped current-voltage behavior is observed in these $\mathrm{NiO}_{\mathrm{x}}$-based devices. It is found that the $\mathrm{S}$-shaped current-voltage behavior is related to the lower dimensional phase distribution and crystallinity at the bottom interface of the RP perovskite layer, and the S-shaped distortion is less severe after the device ageing test.

Keywords: low dimension, perovskite solar cell, S-shaped, high $\mathbf{V}_{\mathrm{oc}}$, phase distribution, hot casting

\section{INTRODUCTION}

Metal-halide perovskites have attracted tremendous attention as revolutionary photovoltaic materials owing to their remarkable electronic and optoelectronic properties such as high absorption coefficient, tunable bandgaps, long carrier diffusion length, and solution-processed and low-cost potential. The inherent instabilities of perovskite are becoming a bottleneck for the practical application of perovskite solar cells (PSCs). Recently, two-dimensional (2D) Ruddlesdon-Popper perovskites (RPPs) have become promising alternatives to 3D perovskite in terms of stability. RPPs are conventionally denoted as $\left(\mathrm{RNH}_{3}\right)_{2} \mathrm{~A}_{\mathrm{n}-1} \mathrm{M}_{\mathrm{n}} \mathrm{X}_{3 \mathrm{n}+1}$, where $\mathrm{RNH}_{3}$ represents bulky aliphatic or aromatic alkylammoniums, for example, butylammonium $\left(\mathrm{BA}^{+}\right)$, iso- $\mathrm{BA}^{+}$, and phenethylammonium $\left(\mathrm{PEA}^{+}\right)$; $\mathrm{A}$ is the cation; $\mathrm{M}$ is the metal ion; and $\mathrm{X}$ is the halide ion. Their hydrophobic organic spacer cations are resistant to the moisture impregnation (Saparov and Mitzi, 2016; Yang et al., 2016; Etgar, 2018). However, 2D RPP perovskites show restricted charge transport, originated from the quantum and confinement effect of 2D RPP perovskites. The improving stability against the ambient environment in 2D RPP perovskites is therefore at the cost of relatively low performance compared to their 3D counterparts. Fortunately, tremendous efforts have been taken to develop high-performance 2D RPP PSCs. Zhang et al (Wu et al., 2019a) reported the 2D $(\mathrm{BA})_{2}(\mathrm{MA})_{3} \mathrm{~Pb}_{4} \mathrm{I}_{13}$ PSCs with the PCE of $17.26 \%$ by developing a slow post-annealing (SPA) process. In 2019, Zhao et al (Ke et al., 2019) broke the PCE records of 2D PSCs by achieving the PCE 
of $18.48 \%$ for $(\mathrm{GA})(\mathrm{MA})_{3} \mathrm{~Pb}_{3} \mathrm{I}_{10}$ perovskite, with improved ambient stabilities. Huang et al. (2021) also reported multifunctional interface engineering by employing guanidinium bromide (GABr) to optimize the secondary crystallization process, realizing a PCE value of $19.3 \%$ for $2 \mathrm{D}$ PSCs under AM 1.5G illumination. The optimized device without encapsulation could retain $94 \%$ of the initial PCE for more than 3,000 $\mathrm{h}$ after being stored under ambient conditions.

Further researches on $2 \mathrm{D}$ perovskite have revealed that different preparation processes for 2D perovskite show significant effect on the crystallization kinetics and the crystal orientation of the resulted $2 \mathrm{D}$ perovskite films. It is widely recognized that crystallization, phase distribution, and orientation profiles of $2 \mathrm{D}$ perovskite have critical influences on charge carrier dynamics and thus the performance of PSCs. Tsai et al. systematically researched hot-casting methods in preparing RP PSCs based on the PEDOT:PSS hole transport layer (HTL) and obtained cells with the PCE of $12.51 \%$ and $\mathrm{V}_{\mathrm{oc}}$ of $\sim 1 \mathrm{~V}$ (Tsai et al., 2016). Meanwhile, the crystal orientation of $2 \mathrm{D}$ perovskite films prepared by hot casting shows a special orientation perpendicular to the glass substrate, which can significantly facilitate efficient charge transport. It has been reported that 2D layered perovskite films cast from perovskite solution precursors with a specific $n$ value are not composed of a single component of n-layered 2D perovskites but rather a mixture of $2 \mathrm{D}$ perovskites with various thicknesses $(n)$ (Quintero-Bermudez et al., 2018). For the hot-casting process, generally speaking, in the obtained 2D perovskite film, the larger $n$ phase is preferentially distributed on the top of the film, which tends to be perpendicular to the substrate; the middle part is multi-n phase; and the small $\mathrm{n}$ phase is more likely distributed on the bottom of the film, which tends to be parallel to the substrate (Xu et al., 2020). This gradient phase distribution in the $2 \mathrm{D}$ perovskite film is conductive to the alignment of the energy bands and facilitates the energy transfer of excitons, and thus the transmission of carriers. Researchers have reported highquality $2 \mathrm{D}$ perovskite films through the hot-casting method, and thus demonstrated high-performance PSCs, but it is worthily noted that most of the common structures reported of inverted quasi-2D RP PSCs are using PEDOT:PSS or PTAA as the hole transport layer (HTL). Unlike the $3 \mathrm{D}$ counterpart, 2D PSCs based on nickel oxide $\left(\mathrm{NiO}_{\mathrm{x}}\right)$ hole transport material are rarely reported, probably due to the S-shaped behavior in the current-voltage characteristics discussed in this work (Chen et al., 2018; Boyd et al., 2020; Liang et al., 2021). Chen et al. fabricated 2D PSCs based on $\mathrm{BA}_{2} \mathrm{MA}_{2} \mathrm{~Pb}_{3} \mathrm{I}_{10}$, and using a $\mathrm{NiO}_{\mathrm{x}} \mathrm{HTL}$ and $\mathrm{a}$ PCBM (50wt\% ICBA) mix ETL. In this structure, 2D PSCs demonstrated a PCE of $12.07 \%$ with a $\mathrm{V}_{\mathrm{oc}}$ of $1.23 \mathrm{~V}$. The author indicated that the decreasing energy loss at the interface between $\mathrm{NiO}_{\mathrm{x}}$ and $n=32 \mathrm{D}$ perovskite was revealed, which attributed to the enhanced $\mathrm{V}_{\mathrm{oc}}$.

Here, we report a hot-casting-processed 2D RP PSC based on $2 \mathrm{D}(\mathrm{BA})_{2}(\mathrm{MA})_{3} \mathrm{~Pb}_{4} \mathrm{I}_{13}(n=4)$ layered perovskite films fabricated with an inverted structure using $\mathrm{NiO}_{x}$ as the hole transport layer (HTL). With the $\mathrm{NiO}_{\mathrm{x}} \mathrm{HTL}$, the RP PSC shows significantly enhanced open-circuit voltage $\left(\mathrm{V}_{\mathrm{oc}}\right)$ compared to devices derived from PEDOT:PSS or PTAA. Our champion device (FTO/ $\left.\mathrm{NiO}_{\mathrm{x}} /(\mathrm{BA})_{2}(\mathrm{MA})_{3} \mathrm{~Pb}_{4} \mathrm{I}_{13} / \mathrm{PCBM} / \mathrm{BCP} / \mathrm{Ag}\right)$ has shown a $\mathrm{V}_{\mathrm{oc}}$ of $1.21 \mathrm{~V}$ under standard AM $1.5 \mathrm{G}$ illumination, which is one of the highest records among recent researches on inverted 2D RPPs PSCs. We observed an S-shaped behavior in the I-V characteristics of our 2D RP PSCs. To investigate the differences of $2 \mathrm{D}$ perovskite films based on different substrates, powder X-ray diffraction (XRD) has been adopted, indicating that the enhanced crystal orientation of $2 \mathrm{D}$ perovskite flakes upon $\mathrm{NiO}_{\mathrm{x}}$-based devices. Spatially resolved photoluminescence (PL) measurements show that a gradient phase distribution from top to bottom is consistent with literature reports, and $\mathrm{NiO}_{\mathrm{x}}$-based devices exhibit a favorable alignment on the multiple perovskite phases and lower trap states of $2 \mathrm{D}$ perovskite flakes. We also performed transient absorption (TA) techniques to compare the charge carrier dynamics in layered perovskite films based on $\mathrm{NiO}_{\mathrm{x}}$ and PEDOT:PSS, further confirming the efficient charge carrier funneling from small-n to large- $\mathrm{n} 2 \mathrm{D}$ phases inside the film, and it is found that a faster charge transfer and suppressed charge recombination occurred in the $\mathrm{NiO}_{\mathrm{x}}$-based devices due to a more enhanced alignment profile of multiple phases of $2 \mathrm{D}$ perovskite component, resulting in a higher $\mathrm{V}_{\mathrm{oc}}$.

\section{RESULTS AND DISCUSSION}

The $2 \mathrm{D}$ perovskite film with a formula of $(\mathrm{BA})_{2}(\mathrm{MA})_{3} \mathrm{~Pb}_{4} \mathrm{I}_{13}$ by using the hot-casting method and subsequent fabrication of inverted PSCs with a structure of $\mathrm{NiO}_{\mathrm{x}}$ or PEDOT:PSS)/ $(\mathrm{BA})_{2}(\mathrm{MA})_{3} \mathrm{~Pb}_{4} \mathrm{I}_{13}$ $/ \mathrm{PCBM} / \mathrm{BCP} / \mathrm{Ag}$, as shown in Figures $\mathbf{1 A}, \mathbf{B}$, shows the comparison of performance of the fabricated 2D RPP perovskite solar cells derived from $\mathrm{NiO}_{\mathrm{x}}$ and PEDOT:PSS. Both devices exhibited relatively high performance relative to those reported records with the similar structure. Intriguingly, via further tests, we observed that the photovoltaic performance of the BA-based 2D PSCs improves after several days storage (ageing) in the glove box, as shown in Figure 1B. After ageing, for the controlled PEDOT:PSS-based devices, we achieved a champion PCE value of $11.0 \%$, a $\mathrm{J}_{\mathrm{sc}}$ of $16.98 \mathrm{~mA} / \mathrm{cm}^{2}$, and $\mathrm{a} \mathrm{V}_{\mathrm{oc}}$ of $0.95 \mathrm{~V}$. The $\mathrm{NiO}_{\mathrm{x}}$-based devices exhibited a remarkable improvement of $\mathrm{V}_{\mathrm{oc}}$ up to $1.2 \mathrm{~V}$ and $\mathrm{J}_{\mathrm{sc}}$ of $17.14 \mathrm{~mA} / \mathrm{cm}^{2}$; as a result, the much improved $\mathrm{V}_{\mathrm{oc}}$ and slightly improved $\mathrm{J}_{\mathrm{sc}}$ led to an overall PCE of $13.7 \%$. Interestingly, it was found that the $\mathrm{J}-\mathrm{V}$ curves of the $\mathrm{NiO}_{\mathrm{x}}$-based device in Figure 1B exhibit an "S" shape around the open-circuit voltage position, resulting in a higher $\mathrm{V}_{\mathrm{oc}}$ value and a relatively lower fill factor (FF). Moreover, we found that this phenomenon occurred in the $\mathrm{NiO}_{\mathrm{x}}$-based PSCs varied during the ageing test, as shown in Figure 2.

In our work, we used the differential coefficient $k$ at the $\mathrm{V}_{\mathrm{oc}}$ point to evaluate the " $S$ "-shaped curve, with a smaller $k$ indicating severer degree of distortion. According to Figure 2, the value of $\mathrm{k} 1$ is summarized at Supplementary Table S1. It is shown that the $k$ value of the corresponding J-V curve increased from 57.93 to 68.97 for the $\mathrm{NiO}_{\mathrm{x}}$-based PSC during the ageing test. The performance of the PSC was enhanced in this process.

Figures 3A-D show the distribution of photovoltaic parameters $\left(\mathrm{V}_{\mathrm{oc}}, \mathrm{J}_{\mathrm{sc}}, \mathrm{FF}\right.$, and $\left.\mathrm{PCE}\right)$ of devices derived from 

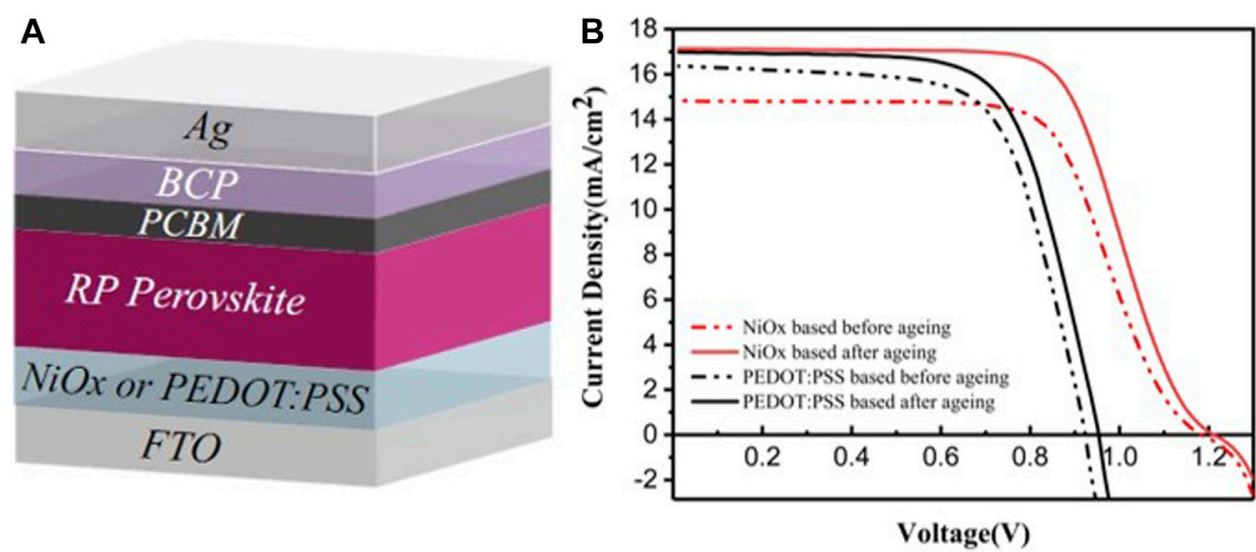

FIGURE 1 | (A) Device architecture of the PSCs. (B) J-V characteristics of device A (NiO ${ }_{x}$-based PSCs) and device B (PEDOT:PSS-based PSCs).

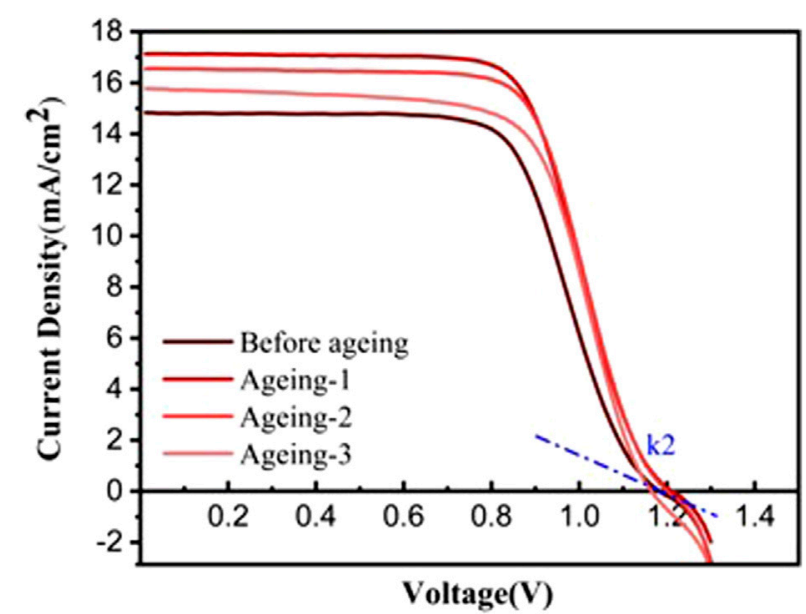

FIGURE 2 | J-V curves of $\mathrm{NiO}_{x}$-based PSCs in different ageing states.

different HTLs. The statistical data were obtained from $\sim 15$ cells. As illustrated in Figure 3, the major improvement lies in the increased $\mathrm{V}_{\mathrm{oc}}$ and more uniform $\mathrm{J}_{\mathrm{sc}}$ and $\mathrm{FF}$ distribution. The external quantum efficiency (EQE) of different HTL devices is shown in Supplementary Figure S1. Note that after the ageing test, the EQE values of $\mathrm{NiO}_{\mathrm{x}}$-based and PEDOT:PSS-based devices are significantly enhanced, matching the promoted short current density in $\mathrm{J}-\mathrm{V}$ curves after ageing, as shown above. The improved $\mathrm{J}_{\mathrm{sc}}$ and EQE could be due to an improved contact between the perovskite and HTLs, the exact mechanism of which requires further investigation. To further the investigation, dark current density-voltage $(\mathrm{J}-\mathrm{V})$ characteristics of different HTL devices before and after ageing was performed, as shown in Supplementary Figure S2. The significant reduction of the leakage current for all samples after ageing is an indication of suppression of carrier recombination (Du et al., 2019).

X-ray diffraction (XRD) measurements were carried out for perovskite films deposited on FTO glass/ $\mathrm{NiO}_{\mathrm{x}}$ and PEDOT:PSS substrates, as illustrated in Supplementary Figure S4. Diffraction peaks at around $14.1^{\circ}$ and $28.3^{\circ}$ in the XRD pattern are ascribed to the (111) and (202) crystallographic planes, respectively. The intensity of the diffraction peaks for both films is consistent with that reported previously on hot-casting methods (Tsai et al., 2016). Furthermore, for the $\mathrm{NiO}_{\mathrm{x}}$-based device, the increase of the intensity for (111) peak is observed, indicating an improved growth quality in (111) direction.

The cross-sectional scanning electron microscopy (SEM) measurements were performed to figure out the effect of the HTL substrate on 2D perovskite films' morphology, as shown in Supplementary Figure S5. $\mathrm{NiO}_{\mathrm{x}^{-}}$and PEDOT:PSS-based samples exhibited a similar smooth and pin-hole free surface, with bricklike grains.

It has been suggested that the phase distribution in $2 \mathrm{D}$ perovskite films plays an important role in the efficiency of photovoltaic devices. We speculated that the substrates with different HTLs may affect the phase formation in 2D layered perovskite films. The perovskite film composition and phase distribution are therefore investigated in order to explain the differences between devices derived from $\mathrm{NiO}_{\mathrm{x}}$ and PEDOT:PSS Supplementary Figure S3 shows light absorption of perovskite layers and different HTLs on FTO. The 2D RP perovskite spin coated on $\mathrm{NiO}_{\mathrm{x}}$ presented slightly higher absorbance than that on the PEDOT:PSS device. Moreover, peaks representing $n=2,3$, and 4 can be observed, which suggests that the 2D RPP perovskite film consists of multiple phases containing different $n$, it is consistent with that reported previously.

The steady-state photoluminescence (PL) spectroscopy measurements were collected with an excitation wavelength of $450 \mathrm{~nm}$ from both the front- (perovskite film side) and back-side (substrate side) illumination to reveal the spatial phase composition of the as-fabricated 2D-RPP films based on different HTLs $\left(\mathrm{NiO}_{\mathrm{x}}\right.$ and PEDOT:PSS). With an excitation wavelength of $450 \mathrm{~nm}$, the penetration depth into layered perovskite films can be estimated to be $\sim 150 \mathrm{~nm}$ based on the absorption spectra, which is smaller than the film thickness of $\sim 350 \mathrm{~nm}$, indicating that the fluorescence signals collected are 

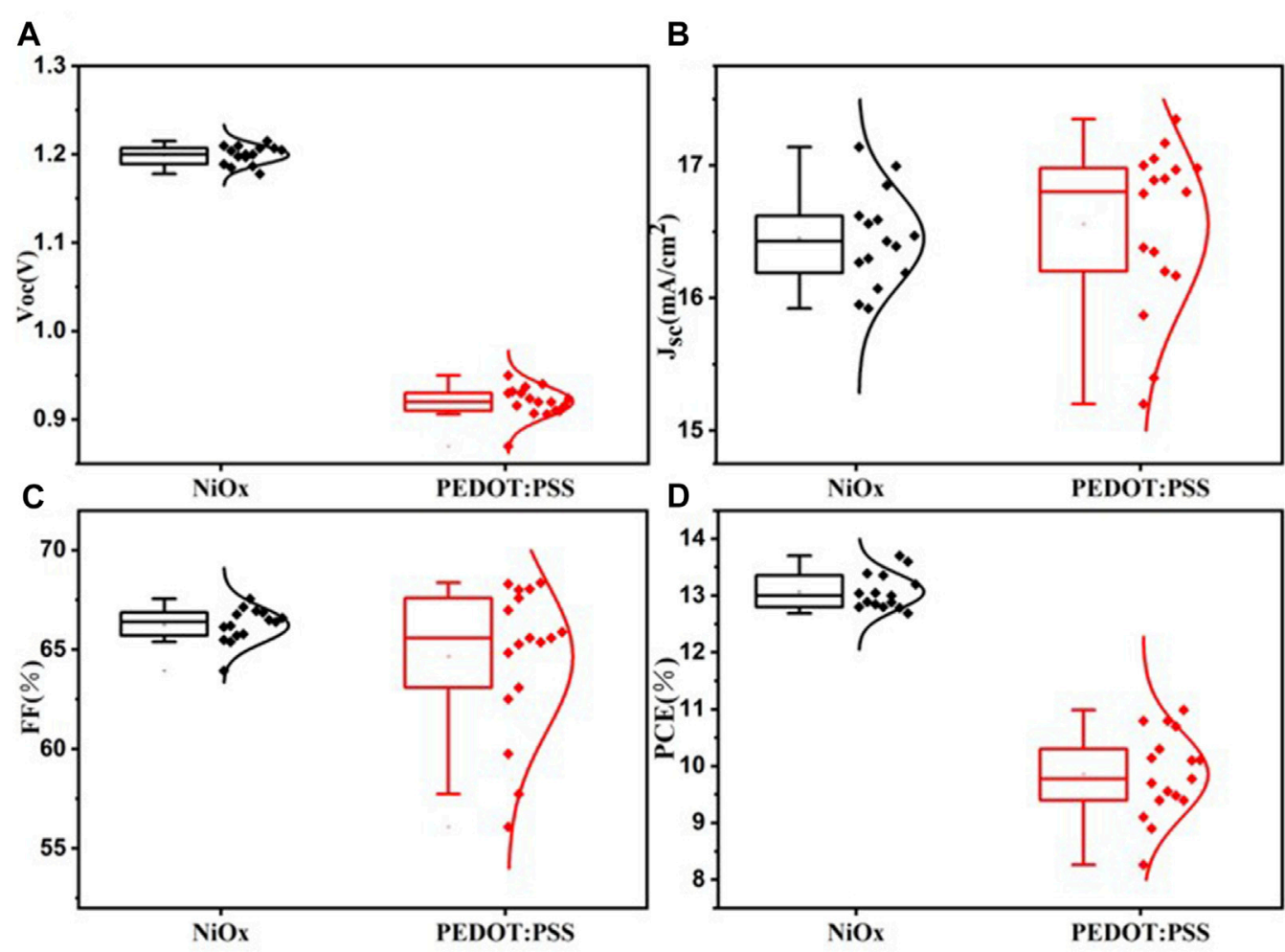

FIGURE 3 |Dependence of photovoltaic parameters of the $\mathrm{NiO}_{x}$ - and PEDOT:PSS-based perovskite devices. The data were statistically analyzed from $~ 15$ cells.

mainly from the same side of films as the photoexcitation (Jang et al., 2020).

In pristine films (before ageing), when excited from the back side (glass side), a dominant long-wavelength PL peak and additional multiple emission peaks at shorter wavelength were detected. According to previous reports, short-wavelength PL peaks in Figure 4A can be assigned to emission of 2D perovskite crystals with small-n phases. The emission peaks at $578 \mathrm{~nm}$, $610 \mathrm{~nm}$, and $640 \mathrm{~nm}$ are corresponding to $n=2,3$, and 4 , respectively. The dominant peak at the long wavelength can be assigned to the $3 \mathrm{D}$-like perovskite. In contrast, as shown in Figure 4B, the PL spectra obtained from the front-side excitation only demonstrated a dominant emission peak at $\sim 740 \mathrm{~nm}$; the dominant peak in Figure $4 B$ at the long wavelength is due to the large-n (3D-like) perovskite component. This result indicates that small-n phases mainly locate at the substrate $\left(\mathrm{NiO}_{\mathrm{x}}\right.$ and PEDOT:PSS) side, and the large-n phases majorly distribute in the upper surface of the films. This phase distribution is beneficial to the self-driven charge separation and transport (from the small-n region to large- $n$ region) in 2D perovskite films (Du et al., 2019). The PL intensity ratio between different emission peaks suggested that the distribution of different phases of layered perovskites was not uniform along the out-of-plane direction in the film. Note that in Figure $4 \mathrm{~B}$, in the front-side excitation, the emission peak of the $\mathrm{NiO}_{\mathrm{x}}$-based sample shifts toward longer wavelength, compared to the PEDOT:PSS-based sample, indicating the improvement of film quality with better crystallization of $3 \mathrm{D}$-like components on the top of the 2D perovskite film (Lian et al., 2019; Meng et al., 2020; Zheng et al., 2020a; Zheng et al., 2020b). Furthermore, the higher PL intensity of emission peaks to the 3D-like component in the $\mathrm{NiO}_{\mathrm{x}}$-based perovskite film from both side excitation indicates the increased crystallinity of 3D-like perovskite phases and the decreased defect state density, compared to PEDOT:PSS-based films (Zheng et al., 2019; Zheng et al., 2020a). However, in Figure 4A, in the back-side excitation, the steady-state $\mathrm{PL}$ is enhanced more strongly in the $\mathrm{NiO}_{\mathrm{x}}$-based film, revealing impeditive hole transmission in the $\mathrm{NiO}_{\mathrm{x}} /$ perovskite surface, and thus causes charge recombination. In addition, compared with the PEDOT:PSS-based perovskite film, it can be found that the intensities of emission peaks at low-n phases considerably enlarge relative to the $3 \mathrm{D}$ phase peak in the $\mathrm{NiO}_{\mathrm{x}}$-based one, indicating the better crystallization and more $2 \mathrm{D}$ phases deposited at the bottom of the $\mathrm{NiO}_{\mathrm{x}}$-based film (Wu et al., 2019a).

We can also find that the PL peaks of different phases enhanced after ageing, as shown in Figure 4, indicating an increased crystallinity for both $2 \mathrm{D}$ and $3 \mathrm{D}$ perovskite phases in the thin film after ageing, and this enhancement is more observable in the $\mathrm{NiO}_{\mathrm{x}}$-based film than in the PEDOT:PSS-based film. This variation of peak intensity of low-n phases in back excitation can be more detectable in Supplementary Figure S6. Moreover, the emission peak position of the large- $n$ perovskite phase in the $\mathrm{NiO}_{\mathrm{x}}$ - and PEDOT:PSS-based perovskite film varied from $\sim 744 \mathrm{~nm}$ and $\sim 741 \mathrm{~nm}$ to $\sim 740 \mathrm{~nm}$ and $\sim 737 \mathrm{~nm}$, respectively. The blue shift of the PL emission resulted from a 

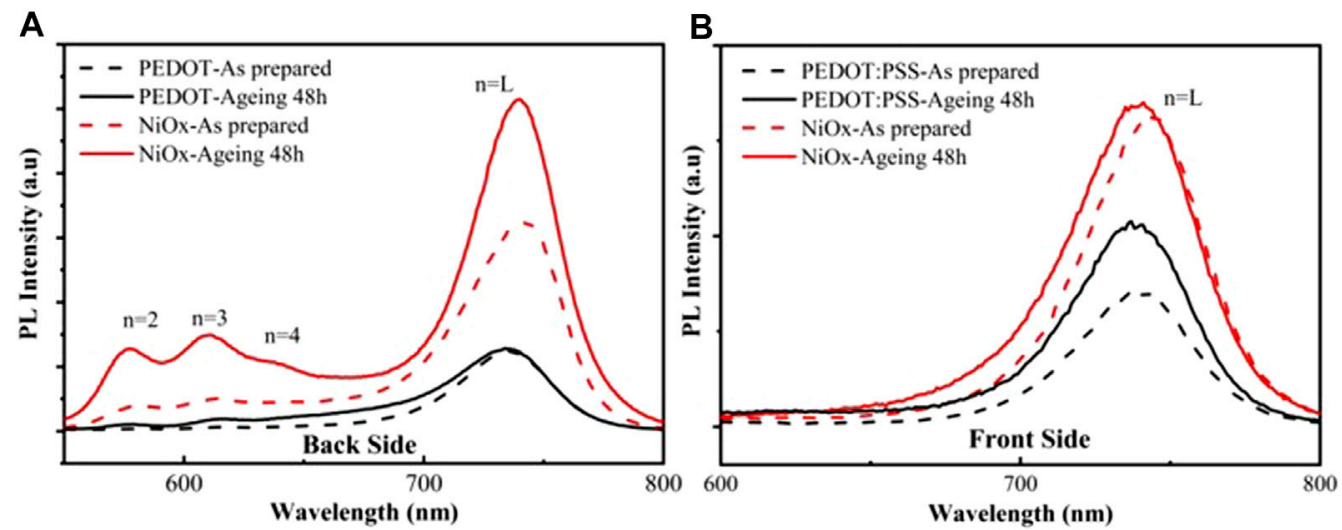

FIGURE 4 | Steady-state PL spectra of films before ageing. (A) Back excitation and (B) front excitation.
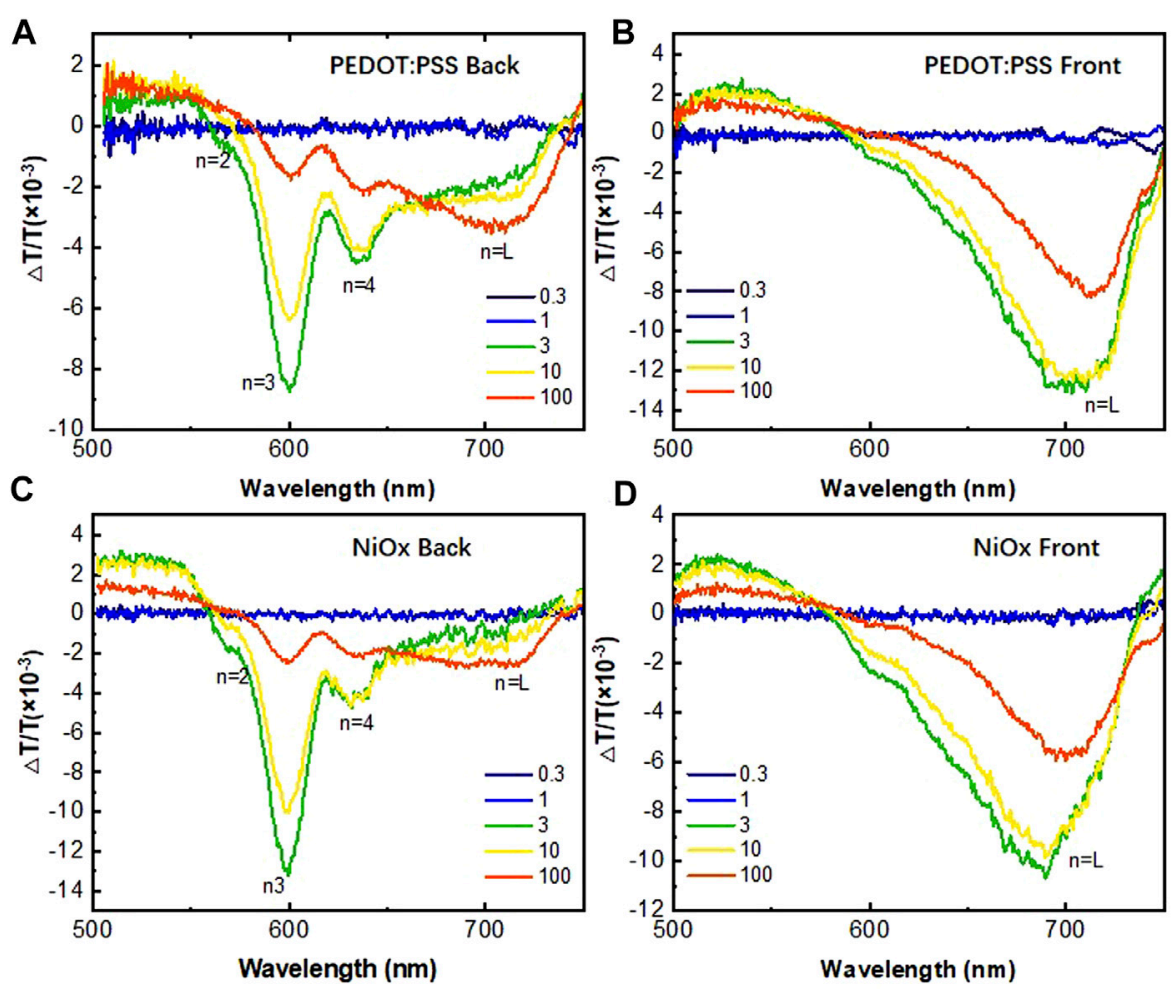

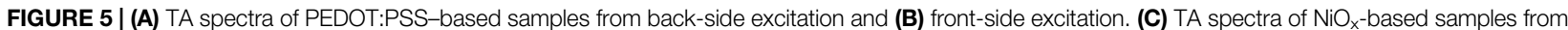
back-side excitation and (D) front-side excitation.

reduced trap density within the $3 \mathrm{D}$-like perovskite after ageing (Chen et al., 2018; Xu et al., 2019; Huang et al., 2021).

Ultrafast transient absorption (TA) measurements were also carried out to further probe the compositional change and carrier dynamic of layered perovskite films upon different substrates, as shown in Figure 6. In order to probe the vertical distribution change of perovskite films, the excitation laser was beamed from the back side (FTO substrate) and from the front side (surface of the perovskite), and the $2 \mathrm{D}$ perovskite films have been ageing for
$160 \mathrm{~h}$, as shown in Figures 5A-D, respectively. In the transient absorption spectra $(\Delta T / T)$, negative signals correspond to ground-state bleaching (GSB). In all cases, a positive weak and broad signal at wavelengths shorter than $600 \mathrm{~nm}$ can be observed, which can be assigned to the derivative feature resulting from the blue shift of the band-edge exciton resonance predominantly from the $n=3$ phase (Wu et al., 2015). Distinct GSB peaks can be easily recognized in the TA spectra. Both the TA spectra of the 2D RPP perovskite film derived from two kinds of HTL devices 

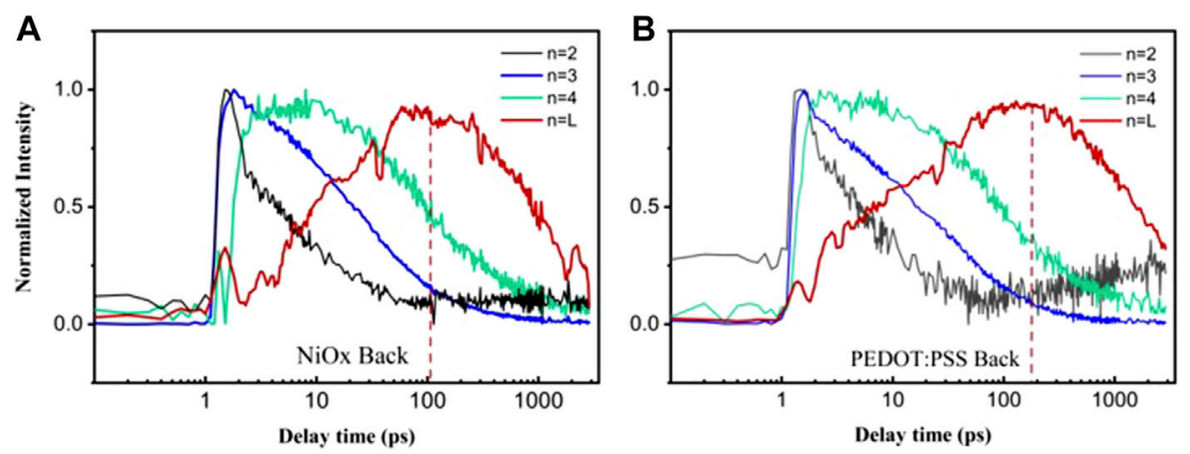

FIGURE 6 | TA kinetics probed at bleach peaks of various $n$ values of the (A) PEDOT:PSS-based film and the (B) $\mathrm{NiO}_{x}$-based film.

exhibit GSB peaks corresponding to phases of $n=2,3,4$, and $\infty$. We can find drastic differences in the TA spectra with opposite excitation, where lower $\mathrm{n}(n=3,4)$ dominated the back-excitation TA spectra. In contrast, with front excitation, GSB of $n=\infty$ becomes significantly stronger. These results further confirm that upon the hot-casting process, perovskite tends to crystallize first on the surface, which leads to $3 \mathrm{D}$ component to preferentially nucleate first on the surface while $2 \mathrm{D}$ components are preferentially segregated at the bottom; this naturally results in the gradient distribution of the QW in the film. This vertical distribution of different perovskite phases allows effective energy transfer from small-n phases to larger-n phases, followed by charge transport to the corresponding electrodes via the cascaded energy levels. It can be found that from the back-site excitation, the intensities of the $n=3$ phase peak are significantly higher and the GSB of the $n=\infty$ phase is weaker in the $\mathrm{NiO}_{\mathrm{x}}$-based film than those in the PEDOT:PSS-based film. It is speculated that films deposited on $\mathrm{NiO}_{\mathrm{x}}$ contain more lower dimension perovskite composition, which is consistent with the PL measurement discussed above, giving a more favorable energy landscape for the faster charge/energy transfer process.

To obtain the transport dynamics of excitons and carriers in layered perovskite films, the TA signals as a function of pumpprobe delay at wavelengths of each GSB peak from back excitation of both samples are plotted in Figure 6. The decay profiles of small- $n$ phases can be attributed to a combined process for exciton decay, including radiative recombination, trap-state filling, and energy transfer to the large-n phase. The rise of the GSB signal of the large-n phase has been previously assigned to the progressive population of excitons in the large-n perovskite phase due to energy transfer from small-n perovskites. Therefore, the GSB signal corresponding to the large- $n$ phase goes on to a decay process on the nanosecond timescale. It is found that the decrease of GSB signals of lower-n phases is followed by GSB peaks at larger-n $(\sim 715 \mathrm{~nm})$. It implies that the sequence in which the cross peaks grow suggests a cascade of energy transfer event from higher energy (low-n) phases to lower energy phases (high-n) in quasi-2D RP perovskite films. Such an energy cascade from a low-n phase to a high-n phase promotes self-driven charge separation and transportation, which is beneficial to the PSC performance (Liu et al., 2017). It can be found that, in Figure 6, the low-energy bleach ( $n=\mathrm{L}$ phase) reaches a maximum magnitude of signal (103.8 ps), which is much faster than that in Figure 6 (185.1 ps), implying faster energy transfer from the low-n phase to the high-n phase.

In the hot-casted quasi-2D RP perovskites, multiple phases of perovskites are distributed in vertical direction from small to large $\mathrm{n}$ and coexist randomly at the bottom of the perovskite thin film (Bube, 1962; Zheng et al., 2019). Lin et al. reported that $2 \mathrm{D}$ perovskite $(\mathrm{BA})_{2}(\mathrm{MA})_{3} \mathrm{~Pb}_{4} \mathrm{I}_{13}$ thin films comprise multiple layered (small-n) perovskite phases surrounded by 3D-like (large-n) phases (Lin et al., 2019). According to this landscape of phase distribution of the 2D RPP perovskite film, excitons and charge carrier transport mechanism have been unveiled (Lin et al., 2020). It is speculated that energy transfer from the larger bandgap layered perovskites to lower bandgap 3D-like perovskites across the ligands at a very early delay timescale. Generally, the layered flakes are few layers in the 2D RP perovskite film, thus most of excited carriers would be transferred to $3 \mathrm{D}$ phase perovskites. After energy transfer, excitons will dissociate into free charge carriers quickly due to the small exciton binding energy in 3D-like perovskite. The collected charge carriers sequentially transport within 3D phase networks with their advantageous high carrier mobilities and long carrier lifetimes. The excitons and charge carrier transport mechanism can be illustrated in Figure 7A.

In the case of the RP 2D phase perovskite, the QW width largely affects the $\mathrm{Eg}$, which is determined by the basic 3D structure and additional quantization due to $2 \mathrm{D}$ confinement of the electron-hole pair. The optical bandgap of the 2D perovskite with general formula $L_{2} A_{n-1} B_{n} X_{3 n+1}$ depends on the value of $n$, and bandgap decreases as the value of $n$ increases due to the quantum and dielectric confinement (Cao et al., 2015; Sichert et al., 2015; Mao et al., 2017; Stoumpos et al., 2017; Gao et al., 2018). For the BA-based RP perovskite, it has been revealed that the optical bandgap increased from 1.52 to $2.24 \mathrm{eV}$ with decreasing $\mathrm{n}$ from 5 to 1 (Blancon et al., 2017). The energy diagram of our PSCs can be illustrated at Figure 7B. The values of the valence band $(\mathrm{VB})$ and conduction band $(\mathrm{CB})$ were adopted from the literature, which were calculated via ultraviolet 

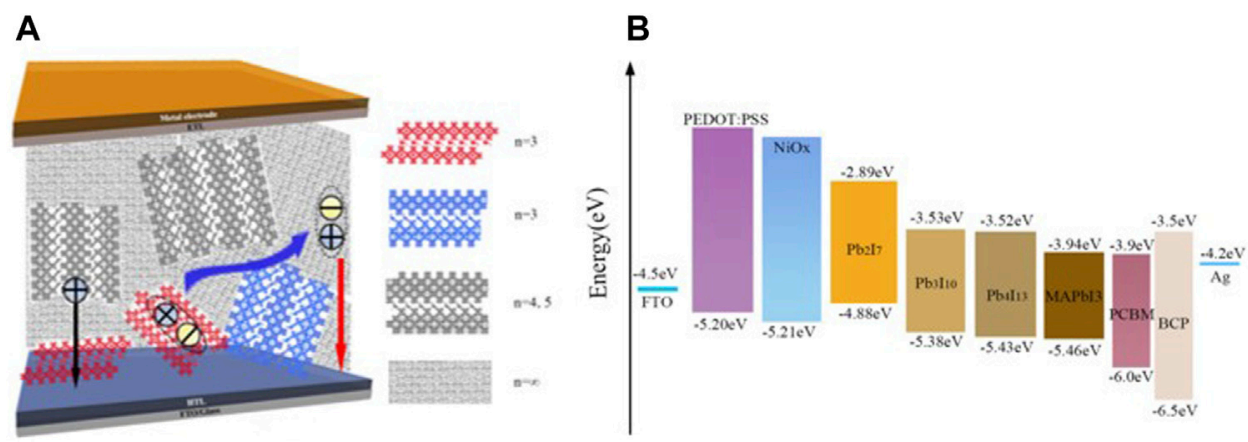

FIGURE 7 | (A) Excitations and charge carrier transport mechanism in the 2D RPP perovskite film. (B) Energy diagram for devices with inverted PSC configuration.

photoelectron spectroscopy (UPS) (Cao et al., 2015; Stoumpos et al., 2016; Liu et al., 2017).

In the above measurement, steady-state PL measurements confirm that a graded vertical phase distributed across the film, where $3 \mathrm{D}$-like phase was preferred to form on the top of the film, whereas low- $n$ phases coexist at the bottom. The $\mathrm{NiO}_{\mathrm{x}}$-based perovskite film shows a higher proportion of low$\mathrm{n}$ phases at the bottom than the PEDOT:PSS-based film. Reports have shown that those $n<3$ perovskite flakes are more likely oriented with their inorganic slabs parallel to the substrate (Shao et al., 2019); in addition, the insulating nature of the chain organic cation and the quantum confinement effect also inhibit the charge transport in the vertical direction, acting as a role of carrier transport barrier (black arrow in Figure 7A), and carriers can only transport to the HTL through the surrounding 3D-like perovskite framework around $2 \mathrm{D}$ flakes in the bottom of the 2D perovskite film (red arrow in Figure 7B) (Lin et al., 2019), resulting in low current density in the resulted PSCs (Figure 1B). After ageing for $48 \mathrm{~h}$, the enhanced PL peaks and the blue shift of the emission peak of 3D-like component indicated better crystallization and lower trap-state density for both $2 \mathrm{D}$ and $3 \mathrm{D}$ phases in $2 \mathrm{D}$ perovskite films, promoting the performance of 2D PSCs.

With further ageing for $160 \mathrm{~h}$, ultrafast transient absorption spectra revealed a faster energy transfer process in the $\mathrm{NiO}_{\mathrm{x}}$-based perovskite film, which can be attributed to a more favorable energy landscape. To sum up, the ageing effect on the 2D RPP perovskite film can be ascribed to enhanced crystallinity and decreased trap-state density. When preparing the $2 \mathrm{D}$ perovskite film with the hot-casting process, due to the preheated substrate, the intermediate between solvent molecules and $\mathrm{PbI}_{2}$ changes quickly and consumes a lot of organic cations in the bottom of the perovskite film, resulting in the gradient distribution of the QW in the film (Xu et al., 2020). After this rapid crystallization process, low-n phases of $2 \mathrm{D}$ perovskite are formed in the bottom of the pristine film with relatively low crystallinity and random orientation (Chen et al., 2019). In terms of the results of PL and TA measurements, we speculated that during the ageing test, with the effect of thermal stress and light soaking, the $2 \mathrm{D}$ perovskite happened to recrystallize gradually, resulting in better crystallinity and preferential orientation.
Consequently, enhanced performance of PSCs was found after the ageing test.

$\mathrm{V}_{\mathrm{oc}}$ is related to the losses via radiative recombination (due to spectral mismatch) and non-radiative recombination involving traps or interfacial misalignment (Wu et al., 2019b). To summarize, the $\mathrm{V}_{\mathrm{oc}}$ enhancement in this work is attributed to a more favorable alignment of multi-perovskite phases in the order of $\mathrm{n}$ values along the vertical direction perpendicular to the substrate in the $\mathrm{NiO}_{\mathrm{x}}$-based RPP perovskite film (Wu et al., 2019a), which can facilitate charge transport with suppressed recombination. The voltage loss via non-radiative recombination is therefore mitigated. The ageing operation can induce better crystallinity and reduced defect density, which facilitate PSC performance.

The S-shaped I-V curves were frequently observed in silicon heterojunction ( $\mathrm{SHJ}$ ) solar cells, $\mathrm{CdTe}$, copper indium (gallium) selenide $[\mathrm{CI}(\mathrm{G}) \mathrm{S}]$, a-Si, and even perovskite cells. It has been suggested that the S-shaped I-V curves were linked to a mismatch between the energy levels of the absorber layer and the charge extraction layers, leading to a barrier for charge extraction ( $\mathrm{Wu}$ et al., 2019b). Moreover, these barriers can be created through a mismatch in band alignment between the absorber layer and the selective contact or the passivation layer. The S-shaped deformation in the $\mathrm{J}-\mathrm{V}$ characteristic is rarely reported in $2 \mathrm{D}$ RPP PSCs. As shown in Figure $\mathbf{7 B}$, the valence band maximum (VBM) of 2D BA-based $n=2 \mathrm{RP}$ perovskites is set at $4.88 \mathrm{eV}$, and the VBM of both $\mathrm{NiO}_{\mathrm{x}}(\sim 5.21 \mathrm{eV})$ and PEDOT:PSS $(\sim 5.2 \mathrm{eV})$ is too deep for holes to be extracted. It is clearly calculated that the energy-level offset $(\triangle \mathrm{E})$ for charge transfer between the VBM of $\mathrm{NiO}_{\mathrm{x}} / \mathrm{PEDOT}$ :PSS and $n=2$ perovskite is $0.32 \mathrm{eV}$, which is of paramount interest to the devices' J-V characteristic (Schulz et al., 2014). Thus, we deduce that the scenario of the $\mathrm{S}$-shaped deformation at the $\mathrm{V}_{\mathrm{oc}}$ point is aroused by the energy-level offset between the $\mathrm{NiO}_{\mathrm{x}}$ and low-dimensional perovskite, causing partial band bending on the surface of $\mathrm{p} / \mathrm{i}$ heterojunction, and thus impeding energy transfer and hole transportation (Aghassi et al., 2018; Li et al., 2018; Saive, 2019; Boyd et al., 2020). According to the results of PL and TA measurements, for $\mathrm{NiO}_{\mathrm{x}}$-based PSCs, with further ageing, 2D components at the bottom of the perovskite film started to recrystallize, forming high crystalline $n=2, n=3$, and $n=4$ phases. The VBM of perovskite further decreased to match the 
VBM of the $\mathrm{NiO}_{\mathrm{x}}$ layer, and thus, the energy barrier for hole transport was reduced, leading to enhanced performance of PSCs, and the S-shaped deformation in J-V curves gradually vanished. These profiles and results are in line with the variation of $\mathrm{J}-\mathrm{V}$ curves and the parameter $k 1$, as shown in Figure $\mathbf{2}$ and Supplementary Table S1.

To sum up, we have fabricated a high $\mathrm{V}_{\mathrm{oc}}(1.2 \mathrm{~V})$ inverted $2 \mathrm{D}$ RP PSCs by the hot-casting method and achieved a power conversion efficiency of $13.7 \%$. Investigations were carried out to explain the difference between devices of two kinds of the HTL. The $\mathrm{NiO}_{\mathrm{x}}$-based 2D layered PSCs possess better phase distribution and crystallinity, and thus resulting in faster charge transmission and lower trap states, resulting in a higher $\mathrm{V}_{\mathrm{oc}}$ than their counterparts. Moreover, a hole transport barrier between the HTL and perovskite surface, which is the cause of the deformation near the $\mathrm{V}_{\mathrm{oc}}$ position, was observed. The origin of this effect needs further investigation; we believed that this observation is beneficial to further evolve the strategies for fabricating large $\mathrm{V}_{\mathrm{oc}}$ and high-performance 2D RP perovskite PSCs.

\section{EXPERIMENTAL METHODS}

\section{Device Fabrication and Characterizations}

Pre-patterned FTO glass plates were sequentially cleaned by ultrasonication in deionized water, acetone, and isopropyl alcohol for $15 \mathrm{~min}$. After $15 \mathrm{~min}$ of UV ozone treatment, different hole transporting layers were deposited on the FTO substrate. For $\mathrm{NiO}_{\mathrm{x}}$-based PSCs, $\mathrm{NiO}_{\mathrm{x}}$ was spin-coated on FTO at $2,000 \mathrm{rpm}$ for $30 \mathrm{~s}$ and annealed in air for $5 \mathrm{~min}$ at $180^{\circ} \mathrm{C}$; this step was repeated for three times, and then, FTO coated with $\mathrm{NiO}_{\mathrm{x}}$ was annealed in air for $2 \mathrm{~h}$ at $480^{\circ} \mathrm{C}$. For PEDOT:PSS-based PSCs, poly(3,4-ethylenedi-oxythiophene):polystyrene sulfonate (PEDOT:PSS) was spin-coated on FTO at 2,000 rotations per minute $(\mathrm{rpm})$ for $60 \mathrm{~s}$ and annealed in air at $150^{\circ} \mathrm{C}$ for $20 \mathrm{~min}$.

\section{REFERENCES}

Aghassi, A., Fay, C. D., and Mozer, A. (2018). Investigation of S-Shaped CurrentVoltage Characteristics in High-Performance Solution-Processed Small Molecule Bulk Heterojunction Solar Cells. Org. Electron. 62, 133-141. doi:10.1016/j.orgel.2018.07.025

Blancon, J.-C., Tsai, H., Nie, W., Stoumpos, C. C., Pedesseau, L., Katan, C., et al. (2017). Extremely Efficient Internal Exciton Dissociation through Edge States in Layered 2D Perovskites. Science 355 (6331), 1288-1292. doi:10.1126/ science.aal4211

Boyd, C. C., Shallcross, R. C., Moot, T., Kerner, R., Bertoluzzi, L., Onno, A., et al. (2020). Overcoming Redox Reactions at Perovskite-Nickel Oxide Interfaces to Boost Voltages in Perovskite Solar Cells. Joule 4 (8), 1759-1775. doi:10.1016/ j.joule.2020.06.004

Bube, R. H. (1962). Trap Density Determination by Space-Charge-Limited Currents. J. Appl. Phys. 33 (5), 1733-1737. doi:10.1063/1.1728818

Cao, D. H., Stoumpos, C. C., Farha, O. K., Hupp, J. T., and Kanatzidis, M. G. (2015). 2D Homologous Perovskites as Light-Absorbing Materials for Solar Cell Applications. J. Am. Chem. Soc. 137 (24), 7843-7850. doi:10.1021/ jacs.5b03796

Chen, A. Z., Shiu, M., Deng, X., Mahmoud, M., Zhang, D., Foley, B. J., et al. (2019). Understanding the Formation of Vertical Orientation in Two-Dimensional
Lately, a 2D RPP perovskite thin film with a formula of (BA) 2(MA)3Pb4I13 was spin-coated on the coated substrate, utilizing the hot-casting process as reported by Tsai et al. (9), and we set our hot-casting temperature at $130^{\circ} \mathrm{C}$. A PCBM electron transporting layer was then sequentially spin-coated on the top of layered perovskite films using PCBM $(20 \mathrm{mg} / \mathrm{ml}$ in chlorobenzene) at 2,000 rpm for $30 \mathrm{~s}$. BCP $(0.5 \mathrm{mg} / \mathrm{ml}$ in ethyl alcohol) was then spin-coated on PCBM at 2,000 rpm for $60 \mathrm{~s}$, followed by deposition of $120 \mathrm{~nm} \mathrm{Ag}$.

\section{DATA AVAILABILITY STATEMENT}

The original contributions presented in the study are included in the article/Supplementary Material; further inquiries can be directed to the corresponding author.

\section{AUTHOR CONTRIBUTIONS}

$\mathrm{HoZ}$ is the dominant researcher, and $\mathrm{HaZ}$ is the adviser.

\section{ACKNOWLEDGMENTS}

This work was financially supported by the Guangdong Natural Science Foundation (2018A030313332) and the Shenzhen Municipal Scientific Program (JCYJ20200109140610435 and ZDSYS201802061805105).

\section{SUPPLEMENTARY MATERIAL}

The Supplementary Material for this article can be found online at: https://www.frontiersin.org/articles/10.3389/fenrg.2021.689657/ full\#supplementary-material
Metal Halide Perovskite Thin Films. Chem. Mater. 31 (4), 1336-1343. doi:10.1021/acs.chemmater.8b04531

Chen, J., Lian, X., Zhang, Y., Yang, W., Li, J., Qin, M., et al. (2018). Interfacial Engineering Enables High Efficiency with a High Open-Circuit Voltage above $1.23 \mathrm{~V}$ in 2D Perovskite Solar Cells. J. Mater. Chem. A. 6 (37), 18010-18017. doi:10.1039/c8ta06925e

Du, X., Qiu, R., Zou, T., Chen, X., Chen, H., and Zhou, H. (2019). Enhanced Uniformity and Stability of Pb-Sn Perovskite Solar Cells via $\mathrm{Me} 4 \mathrm{NBr}$ Passivation. Adv. Mater. Inter. 6 (14), 1900413. doi:10.1002/admi.201900413

Etgar, L. (2018). The merit of Perovskite's Dimensionality; Can This Replace the 3D Halide Perovskite? Energy Environ. Sci. 11 (2), 234-242. doi:10.1039/ c7ee03397d

Gao, P., Bin Mohd Yusoff, A. R., and Nazeeruddin, M. K. (2018). Dimensionality Engineering of Hybrid Halide Perovskite Light Absorbers. Nat. Commun. 9 (1), 5028. doi:10.1038/s41467-018-07382-9

Huang, Y., Li, Y., Lim, E. L., Kong, T., Zhang, Y., Song, J., et al. (2021). Stable Layered 2D Perovskite Solar Cells with an Efficiency of over 19\% via Multifunctional Interfacial Engineering. J. Am. Chem. Soc. 143 (10), 3911-3917. doi:10.1021/jacs.0c13087

Jang, G., Ma, S., Kwon, H.-C., Goh, S., Ban, H., Kim, J. S., et al. (2020). Elucidation of the Formation Mechanism of Highly Oriented Multiphase RuddlesdenPopper Perovskite Solar Cells. ACS Energ. Lett. 6, 249-260. doi:10.1021/ acsenergylett.0c02438 
Ke, W., Mao, L., Stoumpos, C. C., Hoffman, J., Spanopoulos, I., Mohite, A. D., et al. (2019). Compositional and Solvent Engineering in Dion-Jacobson 2D Perovskites Boosts Solar Cell Efficiency and Stability. Adv. Energ. Mater. 9 (10), 1803384. doi:10.1002/aenm.201803384

Li, X., Shen, K., Li, Q., Deng, Y., Zhu, P., and Wang, D. (2018). Roll-over Behavior in Current-Voltage Curve Introduced by an Energy Barrier at the Front Contact in Thin Film CdTe Solar Cell. Solar Energy 165, 27-34. doi:10.1016/ j.solener.2018.02.075

Lian, X., Chen, J., Qin, M., Zhang, Y., Tian, S., Lu, X., et al. (2019). The Second Spacer Cation Assisted Growth of a 2D Perovskite Film with Oriented Large Grain for Highly Efficient and Stable Solar Cells. Angew. Chem. Int. Ed. 58 (28), 9409-9413. doi:10.1002/anie.201902959

Liang, J., Zhang, Z., Zheng, Y., Wu, X., Wang, J., Zhou, Z., et al. (2021). Overcoming Carrier Transport Limitation in Ruddlesden-Popper Perovskite Films by Lamellar Nickel Oxide Substrate. J. Mater. Chem. A 89, 11741-11752. doi:10.1039/d1ta01038g

Lin, D., Ma, L., Ni, W., Wang, C., Zhang, F., Dong, H., et al. (2020). Unveiling Hot Carrier Relaxation and Carrier Transport Mechanisms in Quasi-TwoDimensional Layered Perovskites. J. Mater. Chem. A. 8 (47), 25402-25410. doi:10.1039/d0ta09530c

Lin, Y., Fang, Y., Zhao, J., Shao, Y., Stuard, S. J., Nahid, M. M., et al. (2019). Unveiling the Operation Mechanism of Layered Perovskite Solar Cells. Nat. Commun. 10 (1), 1008. doi:10.1038/s41467-019-08958-9

Liu, J., Leng, J., Wu, K., Zhang, J., and Jin, S. (2017). Observation of Internal Photoinduced Electron and Hole Separation in Hybrid Two-Dimentional Perovskite Films. J. Am. Chem. Soc. 139 (4), 1432-1435. doi:10.1021/jacs.6b12581

Mao, L., Wu, Y., Stoumpos, C. C., Traore, B., Katan, C., Even, J., et al. (2017). Tunable White-Light Emission in Single-Cation-Templated Three-Layered 2D Perovskites (CH3CH2NH3)4Pb3Br10-xClx. J. Am. Chem. Soc. 139 (34), 11956-11963. doi:10.1021/jacs.7b06143

Meng, J., Song, D., Huang, D., Li, Y., Li, Y., Maqsood, A., et al. (2020). Enhanced VOC of Two-Dimensional Ruddlesden-Popper Perovskite Solar Cells Using Binary Synergetic Organic Spacer Cations. Phys. Chem. Chem. Phys. 22 (1), 54-61. doi:10.1039/c9cp04018h

Quintero-Bermudez, R., Gold-Parker, A., Proppe, A. H., Munir, R., Yang, Z., Kelley, S. O., et al. (2018). Compositional and Orientational Control in Metal Halide Perovskites of Reduced Dimensionality. Nat. Mater 17 (10), 900-907. doi:10.1038/s41563-018-0154-x

Saive, R. (2019). S-shaped Current-Voltage Characteristics in Solar Cells: A Review. IEEE J. Photovoltaics 9 (6), 1477-1484. doi:10.1109/jphotov.2019.2930409

Saparov, B., and Mitzi, D. B. (2016). Organic-Inorganic Perovskites: Structural Versatility for Functional Materials Design. Chem. Rev. 116 (7), 4558-4596. doi:10.1021/acs.chemrev.5b00715

Schulz, P., Edri, E., Kirmayer, S., Hodes, G., Cahen, D., and Kahn, A. (2014). Interface Energetics in Organo-Metal Halide Perovskite-Based Photovoltaic Cells. Energ. Environ. Sci. 7 (4), 1377. doi:10.1039/c4ee00168k

Shao, S., Duim, H., Wang, Q., Xu, B., Dong, J., Adjokatse, S., et al. (2019). Tuning the Energetic Landscape of Ruddlesden-Popper Perovskite Films for Efficient Solar Cells. ACS Energ. Lett. 5 (1), 39-46. doi:10.1021/acsenergylett.9b02397

Sichert, J. A., Tong, Y., Mutz, N., Vollmer, M., Fischer, S., Milowska, K. Z., et al. (2015). Quantum Size Effect in Organometal Halide Perovskite Nanoplatelets. Nano Lett. 15 (10), 6521-6527. doi:10.1021/acs.nanolett.5b02985

Stoumpos, C. C., Cao, D. H., Clark, D. J., Young, J., Rondinelli, J. M., Jang, J. I., et al. (2016). Ruddlesden-Popper Hybrid Lead Iodide Perovskite 2D Homologous
Semiconductors. Chem. Mater. 28 (8), 2852-2867. doi:10.1021/ acs.chemmater.6b00847

Stoumpos, C. C., Soe, C. M. M., Tsai, H., Nie, W., Blancon, J.-C., Cao, D. H., et al. (2017). High Members of the 2D Ruddlesden-Popper Halide Perovskites: Synthesis, Optical Properties, and Solar Cells of (CH3(CH2)3NH3)2(CH3NH3)4Pb5I16. Chem 2 (3), 427-440. doi:10.1016/j.chempr.2017.02.004

Tsai, H., Nie, W., Blancon, J.-C., Stoumpos, C. C., Asadpour, R., Harutyunyan, B., et al. (2016). High-efficiency Two-Dimensional Ruddlesden-Popper Perovskite Solar Cells. Nature 536 (7616), 312-316. doi:10.1038/nature18306

Wu, G., Li, X., Zhou, J., Zhang, J., Zhang, X., Leng, X., et al. (2019a). Fine Multiphase Alignments in 2D Perovskite Solar Cells with Efficiency over $17 \%$ via Slow Post-Annealing. Adv. Mater. 31 (42), e1903889. doi:10.1002/ adma.201903889

Wu, G., Zhou, J., Zhang, J., Meng, R., Wang, B., Xue, B., et al. (2019b). Management of the Crystallization in Two-Dimensional Perovskite Solar Cells with Enhanced Efficiency within a Wide Temperature Range and High Stability. Nano Energy 58, 706-714. doi:10.1016/j.nanoen.2019.02.002

Wu, X., Trinh, M. T., and Zhu, X.-Y. (2015). Excitonic Many-Body Interactions in Two-Dimensional Lead Iodide Perovskite Quantum Wells. J. Phys. Chem. C 119 (26), 14714-14721. doi:10.1021/acs.jpcc.5b00148

Xu, Q., Meng, K., Liu, Z., Wang, X., Hu, Y., Qiao, Z., et al. (2019). Synergistic Improvements in Efficiency and Stability of 2D Perovskite Solar Cells with Metal Ion Doping. Adv. Mater. Inter. 6 (23), 1901259. doi:10.1002/ admi.201901259

Xu, Y., Wang, M., Lei, Y., Ci, Z., and Jin, Z. (2020). Crystallization Kinetics in 2D Perovskite Solar Cells. Adv. Energ. Mater. 10, 2002558. doi:10.1002/ aenm.202002558

Yang, S., Wang, Y., Liu, P., Cheng, Y.-B., Zhao, H. J., and Yang, H. G. (2016). Functionalization of Perovskite Thin Films with Moisture-Tolerant Molecules. Nat. Energ. 1 (2). doi:10.1038/nenergy.2015.16

Zheng, F., Chen, W., Bu, T., Ghiggino, K. P., Huang, F., Cheng, Y., et al. (2019). Triggering the Passivation Effect of Potassium Doping in Mixed-Cation MixedHalide Perovskite by Light Illumination. Adv. Energ. Mater. 9 (24), 1901016. doi:10.1002/aenm.201901016

Zheng, F., Zuo, C., Niu, M., Zhou, C., Bradley, S. J., Hall, C. R., et al. (2020a). Revealing the Role of Methylammonium Chloride for Improving the Performance of 2D Perovskite Solar Cells. ACS Appl. Mater. Inter. 12 (23), 25980-25990. doi:10.1021/acsami.0c05714

Zheng, H. L., Liu, D. T., Wang, Y. F., Yang, Y. G., Li, H., Zhang, T., et al. (2020b). Synergistic Effect of Additives on 2D Perovskite Film towards Efficient and Stable Solar Cell. Chem. Eng. J. 389, 9. doi:10.1016/j.cej.2020.124266

Conflict of Interest: The authors declare that the research was conducted in the absence of any commercial or financial relationships that could be construed as a potential conflict of interest.

Copyright @ 2021 Zhong, Zhou, Wu, Lin, Wang, Li and Zhou. This is an open-access article distributed under the terms of the Creative Commons Attribution License (CC $B Y)$. The use, distribution or reproduction in other forums is permitted, provided the original author(s) and the copyright owner(s) are credited and that the original publication in this journal is cited, in accordance with accepted academic practice. No use, distribution or reproduction is permitted which does not comply with these terms. 